\title{
Harmonically trapped Fermi gas: Temperature dependence of the Tan contact
}

\author{
Yangqian Yan $^{1}$ and D. Blume ${ }^{1,2}$ \\ ${ }^{1}$ Department of Physics and Astronomy, Washington State University, Pullman, Washington 99164-2814, USA \\ ${ }^{2}$ ITAMP, Harvard-Smithsonian Center for Astrophysics, Cambridge, Massachusetts 02138, USA
}

(Dated: July 19, 2018)

\begin{abstract}
Ultracold atomic gases with short-range interactions are characterized by a number of universal species-independent relations. Many of these relations involve the two-body Tan contact. Employing the canonical ensemble, we determine the Tan contact for small harmonically trapped twocomponent Fermi gases at unitarity over a wide range of temperatures, including the zero and high temperature regimes. A cluster expansion that describes the properties of the $N$-particle system in terms of those of smaller subsystems is introduced and shown to provide an accurate description of the contact in the high temperature regime. Finite-range corrections are quantified and the role of the Fermi statistics is elucidated by comparing results for Fermi, Bose and Boltzmann statistics.
\end{abstract}

PACS numbers:

Systems with short-range interactions are characterized by universal relations that are independent of the details of the underlying interactions. The Tan contact [1 5], e.g., enters into a large number of universal relations and connects physically distinct quantities such as the large momentum tail, the inelastic loss rate, the number of pairs with small interparticle distances, and certain characteristics of radio frequency (rf) spectra. A striking feature of many of the universal relations is that they apply to homogeneous and inhomogeneous systems at zero and finite temperature.

Yet, although many universal relations that evolve around the Tan contact are known, only a few explicit measurements [6-10] and calculations [11 18] of the Tan contact exist. At present, the dependence of the Tan contact of the spin-balanced homogeneous two-component Fermi gas at unitarity on the temperature is highly debated. While experimental data [9], extracted from the rf lineshape of a potassium mixture in two different hyperfine states, indicate a fairly sharp jump of the contact around the superfluid transition temperature, two sets of Monte Carlo simulations [14, 15] - which disagree with each other - do not seem to see this jump.

This paper considers small Fermi gases consisting of $N_{1}$ spin-up and $N_{2}$ spin-down fermions under external spherically symmetric harmonic confinement. Working in the canonical ensemble, we determine the Tan contact at unitarity with an accuracy at the few percent level as a function of the temperature, including the low (near zero) temperature regime and the high temperature regime. Our main findings are: (i) The high temperature tail of the contact $C_{N_{1}, N_{2}}$ is well approximated by the contacts of the $\left(N_{1}^{\prime}, N_{2}^{\prime}\right)$ subclusters with $N_{1}^{\prime}+N_{2}^{\prime}<N$, where $N_{1}^{\prime} \leq N_{1}$ and $N_{2}^{\prime} \leq N_{2}$. The connection between this cluster expansion, which can be applied to any thermodynamic observable calculated in the canonical ensemble, and the virial expansion that has recently been applied extensively to determine thermodynamic properties of atomic gases in the grand canonical ensemble 19
21], is described. (ii) While the contact of the trapped $\left(N_{1}, N_{2}\right)=(1,1)$ and $(2,2)$ systems is maximal at $T=0$, that of the $(2,1),(3,1)$ and $(4,1)$ systems shows a maximum at finite temperature. A microscopic interpretation of this behavior is offered. (iii) For the cases studied, the contact shows a non-negligible dependence on the range $r_{0}$ of the underlying two-body potential at low temperature; in the high temperature regime, in contrast, the range dependence is negligible. (iv) Fermi statistics plays a role at temperatures where three-body physics is nonnegligible. The role of the Fermi statistics is elucidated by turning the exchange symmetry off and by switching to Bose statistics.

The two-component Fermi gas consisting of $N$ atoms with mass $m$ and position vectors $\mathbf{r}_{j}(j=1, \cdots, N)$ is described by the model Hamiltonian $H$,

$$
H=\sum_{j=1}^{N}\left(\frac{-\hbar^{2}}{2 m} \nabla_{j}^{2}+\frac{1}{2} m \omega^{2} \mathbf{r}_{j}^{2}\right)+\sum_{j=1}^{N_{1}} \sum_{k=N_{1}+1}^{N} V_{\mathrm{tb}}\left(\mathbf{r}_{j k}\right),
$$

where $\omega$ denotes the angular trapping frequency. We consider two different interspecies two-body potentials $V_{\mathrm{tb}}$, a regularized zero-range pseudopotential $V_{\mathrm{F}}$ 22. and a short-range Gaussian potential $V_{\mathrm{G}}$ with depth $U_{0}$ $\left(U_{0}<0\right)$ and range $r_{0}, V_{\mathrm{G}}\left(\mathbf{r}_{j k}\right)=U_{0} \exp \left[-\mathbf{r}_{j k}^{2} /\left(2 r_{0}^{2}\right)\right]$. For a given $r_{0}$, we adjust $U_{0}$ such that $V_{\mathrm{G}}$ supports a single zero-energy $s$-wave bound state in free space, i.e., such that the $s$-wave scattering length $a_{s}$ diverges. Our calculations use $r_{0} \ll a_{\mathrm{ho}}$, where $a_{\mathrm{ho}}$ is the harmonic oscillator length, $a_{\mathrm{ho}}=\sqrt{\hbar /(m \omega)}$. This Letter considers temperatures ranging from $T=0$ to $k_{B} T \gg E_{\mathrm{ho}}$, where $E_{\mathrm{ho}}=\hbar \omega$. The largest temperatures considered are chosen such that the two-body interactions can be reliably parametrized by the $s$-wave scattering length and corresponding effective range correction, i.e., so that higher partial wave contributions in the two-body sector can be neglected.

To determine the Tan contact $C_{N_{1}, N_{2}}$, we employ the 
adiabatic and pair relations,

$$
C_{N_{1}, N_{2}}=\frac{4 \pi m}{\hbar^{2}}\left\langle\frac{\partial E\left(a_{s}\right)}{\partial\left(-a_{s}^{-1}\right)}\right\rangle_{\mathrm{th}}
$$

and

$$
C_{N_{1}, N_{2}}=4 \pi \lim _{s \rightarrow 0} \frac{\left\langle N_{\text {pair }}^{r<s}\right\rangle_{\text {th }}}{s}
$$

here, the $\langle\cdot\rangle_{\text {th }}$ notation indicates a thermal average and $E\left(a_{s}\right)$ denotes the energy of the system. The quantity $N_{\text {pair }}^{r<s}$ is the number of pairs with interspecies distances smaller than $s$. For zero-range interactions, $s$ is taken to zero. For finite-range interactions, in contrast, $s$ goes to a small value such that $s$ is larger than the range $r_{0}$ of the underlying two-body potential. The pair relation can be written in terms of the short distance behavior of the pair distribution function $P_{12}(r)$ for the spin-up - spin-down pairs [1 3, 11]. Throughout, we employ the normalization $4 \pi \int_{0}^{\infty} P_{12}(r) r^{2} d r=N_{1} N_{2}$. The thermally averaged expectation values are obtained by employing two complementary approaches, a "microscopic approach" and a "direct approach".

In the microscopic approach, the thermal expectation value of an observable $A$ is obtained using $\langle A\rangle_{\mathrm{th}}=\sum_{j} \exp \left[-E_{j} /\left(k_{B} T\right)\right] A_{j} / \sum_{j} \exp \left[-E_{j} /\left(k_{B} T\right)\right]$, where the sum runs over all eigen energies $E_{j}$ (with associated eigen states $\psi_{j}$ ) of the Hamiltonian $H$ and $A_{j}=\left\langle\psi_{j}|A| \psi_{j}\right\rangle /\left\langle\psi_{j} \mid \psi_{j}\right\rangle$. The solutions to the timeindependent Schrödinger equation are obtained semianalytically for the interaction model $V_{\mathrm{F}}$ 23, 24 and using a basis set expansion approach for the interaction model $V_{\mathrm{G}}$ [25 27]. The direct approach is based on calculating $\langle A\rangle$ from the density matrix $\rho,\langle A\rangle=$ $\operatorname{Tr}(A \rho) / \operatorname{Tr}(\rho)$. To sample $\rho$, we employ the path integral Monte Carlo (PIMC) approach [28]. Because of the Fermi sign problem [29], the applicability of this approach is expected to be limited to the high temperature regime.

Figure 1(a) shows the scaled pair distribution function $r^{2} P_{12}(r)$ for the $(3,1)$ system for four temperatures, $k_{B} T / E_{\mathrm{ho}}=0,0.6,1.2$ and 2 . At $T=0$ [dotted line in Fig. [1(a)], $P_{12}$ is governed by the lowest eigenstate, which has $L^{\Pi}=1^{+}$symmetry [27] ( $L$ and $\Pi$ denote the orbital angular momentum and parity, respectively). As the temperature increases, excited states contribute. The second lowest state has $1^{-}$symmetry. Compared to the ground state, its $P_{12}$ has an increased amplitude in the small but finite $r$ region. The scaled pair distribution function $r^{2} P_{12}$ for $k_{B} T=0.6 E_{\mathrm{ho}}$ (dashed line) has a comparable amplitude to that for $T=0$; however, clear differences are visible at larger interspecies distances $r$. For yet larger $T$, the small $r$ amplitude decreases drastically [see dash-dotted and dash-dot-dotted lines in Fig. 1(a)] while the maximum of $r^{2} P_{12}$ moves to larger $r$. To extract the contact from $r^{2} P_{12}$, we fit the small $r$ region $(r$

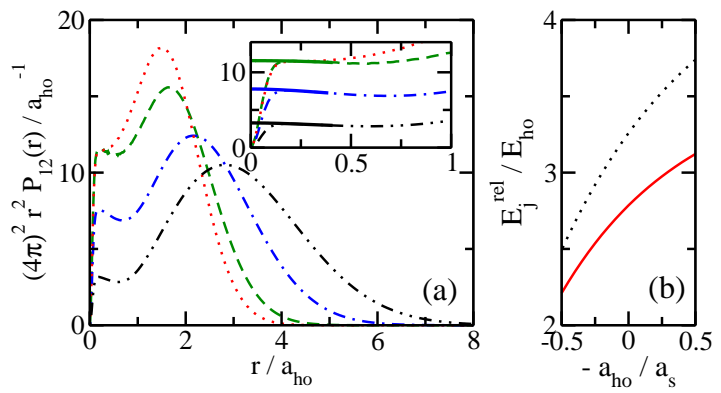

FIG. 1: (Color online) (a) The dotted, dashed, dash-dotted and dash-dot-dotted lines show the scaled pair distribution function $r^{2} P_{12}(r)$ for $k_{B} T /(\hbar \omega)=0,0.6,1.2$ and 2 , respectively, for the $(3,1)$ system interacting through $V_{\mathrm{G}}$ with $r_{0}=0.06 a_{\mathrm{ho}}$. The $T=0$ curve is determined using the basis set expansion approach while the finite $T$ curves are determined using the PIMC approach. The thick solid lines in the inset of panel (a), which is a blow-up of the small $r$ region, show the extrapolation to $r=0$. (b) The solid and dotted lines show the relative energy of the ground state with $L^{\Pi}=1^{-}$and first excited state with $L^{\Pi}=0^{+}$of the $(2,1)$ system interacting through $V_{\mathrm{G}}$ with $r_{0}=0.06 a_{\mathrm{ho}}$ as a function of $-1 / a_{s}$.

larger than $r_{0}$ ) and extrapolate the fit to $r=0$ [see thick solid lines in the inset of Fig. 1(a)].

Figure2 2 shows the contact $C_{N_{1}, N_{2}}$ at unitarity for $N=$ $2-4$ as a function of the temperature. The symbols show the PIMC results, obtained by analyzing the scaled pair distribution functions $r^{2} P_{12}(r)$ for $V_{\mathrm{G}}$ with $r_{0} \ll$ $a_{\text {ho }}$. The solid lines in Fig. 2 show the contact for $r_{0}=$ $0.06 a_{\text {ho }}$ obtained by evaluating the adiabatic relation via the microscopic approach. It can be seen that the contact calculated by evaluating the adiabatic relation via the microscopic approach and the pair relation via the direct approach agree or connect smoothly for the three system sizes considered.

To estimate the dependence of the contact on the range $r_{0}$ of the underlying two-body potential, we determine the contact of the $(1,1)$ and $(2,1)$ systems with zerorange interactions (see supplemental material). The dotted lines in Figs. 2(a) and 2(b) show the result. In the low temperature regime, the contact for $r_{0}=0$ lies below that for $r_{0}>0$ for the $(1,1)$ and $(2,1)$ systems. At $k_{B} T=0.4 E_{\mathrm{ho}}$, e.g., the $(1,1)$ and $(2,1)$ contacts for $r_{0}=0.06 a_{\text {ho }}$ lie $1.5 \%$ and $3 \%$, respectively, above the contact for $r_{0}=0$. At large $T$, the dependence of the contact on the range is negligibly small. Our PIMC simulations suggest a similar range dependence for the $(3,1)$, $(4,1)$ and $(2,2)$ systems.

Figures 2(a)-2(d) show an intriguing dependence of the contact on the temperature. $C_{1,1}$ and $C_{2,2}$ decrease monotonically with increasing temperature while $C_{2,1}$ and $C_{3,1}$ exhibit a maximum at $k_{B} T \approx 0.36 E_{\mathrm{ho}}$ and between $0.4 E_{\mathrm{ho}}$ and $0.5 E_{\mathrm{ho}}$, respectively. To explain this behavior, it is instructive to evaluate the adiabatic rela- 


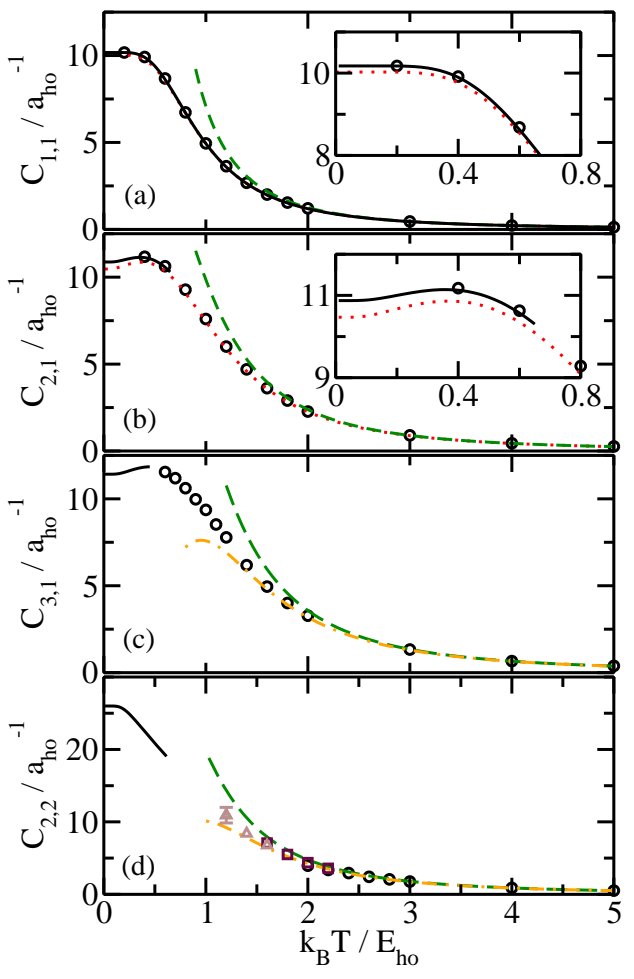

FIG. 2: (Color online) Contact $C_{N_{1}, N_{2}}$ as a function of $k_{B} T / E_{\text {ho }}$ for the (a) $(1,1),(\mathrm{b})(2,1)$, (c) $(3,1)$, and (d) $(2,2)$ systems. The circles, squares and triangles show $C_{N_{1}, N_{2}}$ for $V_{\mathrm{G}}$ with $r_{0} / a_{\mathrm{ho}}=0.06,0.08$ and 0.1 obtained using the PIMC approach. The solid lines show $C_{N_{1}, N_{2}}$ for $V_{\mathrm{G}}$ with $r_{0}=0.06 a_{\text {ho }}$ obtained using the basis set expansion approach. For comparison, the dotted lines in panels (a) and (b) show $C_{N_{1}, N_{2}}$ obtained using $V_{\mathrm{F}}$. (a) The dashed line shows the first-order Taylor expansion at high temperature. (b) The dashed line shows the cluster expansion, i.e., the quantity $2 C_{1,1}$. (c)/(d) The dashed and dash-dotted lines show the leading order term of the cluster expansion and the full cluster expansion, respectively. The insets of panels (a) and (b) show blow-ups of the low temperature regions.

tion via the microscopic approach.

For the $(1,1)$ system with zero-range interactions, one finds $\partial E_{j} /\left(\partial\left(-a_{s}^{-1}\right)\right)=\Gamma(j+1 / 2) 2^{3 / 2} /[\pi(2 j) !] E_{\mathrm{ho}} a_{\mathrm{ho}}$ for the $s$-wave states and $\partial E_{j} /\left(\partial\left(-a_{s}^{-1}\right)\right)=0$ for all higher partial wave states [23, 30]. The fact that $C_{1,1}$ decreases monotonically with decreasing temperature is thus a direct consequence of the fact that $\partial E_{j} /\left(\partial\left(-a_{s}^{-1}\right)\right)$ (for $s$-wave states) decreases with increasing $j$. The inclusion of effective range corrections does not, if applied to the Gaussian model interaction with sufficiently small $r_{0}$, change this picture [31]. A similar analysis, based on the numerically determined energies, holds for the $(2,2)$ system. Figure 1(b) shows the lowest two relative eigen energies, which correspond to states with $L^{\Pi}=1^{-}$and $0^{+}$symmetry, respectively, of the $(2,1)$ system as a function of $-a_{\mathrm{ho}} / a_{s}$ for $r_{0}=0.06 a_{\mathrm{ho}}$. The slope of the $1^{-}$ state is smaller than that of the $0^{+}$state. This can be understood as follows. In the $a_{s} \rightarrow 0^{-}$limit, the lowest state has $L^{\Pi}=1^{-}$symmetry. In the $a_{s} \rightarrow 0^{+}$limit, in contrast, the lowest state has $L^{\Pi}=0^{+}$symmetry. The two states cross at $\left.a_{\mathrm{ho}} / a_{s} \approx 1 \quad 32-34\right]$. Correspondingly, in the unitary regime the energy of the lowest $L=0$ state changes more rapidly with $-1 / a_{s}$ than that of the lowest $L=1$ state, implying that the contact at unitarity increases in the low temperature regime where the inclusion of only two states yields converged results. A more comprehensive analysis that accounts for all states is presented in the supplemental material [31]. For the $(3,1)$ system, a similar argument can be made in the low temperature regime where the inclusion of just a few states suffices. The calculations presented here suggest that $C_{N_{1}, N_{2}}$ decreases monotonically with $T$ if $N_{1}-N_{2}=0$ and exhibits a maximum at finite $T$ if $N_{1}-N_{2} \neq 0$. While it is tempting to generalize these results to larger systems, it should be noted that the density of states increases dramatically with increasing $N$ and that application of a few-state model will be limited to smaller temperatures as $N$ increases.

We now introduce a high temperature cluster expansion of the contact at unitarity. A formal discussion of the cluster expansion in the canonical ensemble applied to classical systems is provided in Ref. [35]. The $\left(N_{1}, N_{2}\right)$ system contains $N_{1} N_{2}$ interacting pairs and one might expect that, using the argument that twocomponent Fermi gases behave universally [36, 37], the high temperature tail of $C_{N_{1}, N_{2}}$ is governed by $N_{1} N_{2} C_{1,1}$ for $k_{B} T \gg E_{\mathrm{ho}}$ [see dashed lines in Figs. 2(b)-2(d)]. The next term in the cluster expansion, applicable to systems with $N>3$, depends on the "three-body term" $C_{2,1}-2 C_{1,1}$,

$$
\frac{C_{N_{1}, N_{2}}}{N_{1} N_{2}}=C_{1,1}+\frac{N_{1}+N_{2}-2}{2}\left(C_{2,1}-2 C_{1,1}\right)+\cdots .
$$

The dashed and dash-dotted lines in Figs. 2(c) and 2(d) show the leading term and the sum of the leading and sub-leading terms for the $(3,1)$ and $(2,2)$ systems. The inclusion of the three-body term improves the validity regime of the cluster expansion notably. Assuming zero-range interactions, the leading order terms of the Taylor expansions of $C_{1,1}$ and $C_{2,1}-2 C_{1,1}$ around $\tilde{\omega} \ll 1$, where $\tilde{\omega}=E_{\mathrm{ho}} /\left(k_{B} T\right)$, are $4 \pi^{1 / 2} \tilde{\omega}^{5 / 2} a_{\mathrm{ho}}^{-1}$ and $-7.012(12) \tilde{\omega}^{11 / 2} a_{\mathrm{ho}}^{-1}$, indicating that the three-body term is suppressed by $\tilde{\omega}^{3}$ compared to the leading order twobody term. Figures 2(c) and 2(d) show that the numerically obtained $C_{3,1}$ and $C_{2,2}$ contacts (symbols) lie above the cluster prediction (dash-dotted line), suggesting that the corresponding leading order four-body expansion coefficients are positive. The above expansions can be viewed as canonical analogs of the virial equation of state description of the contact within the grand canonical ensemble [21, 31, 38].

Equation (4) shows that the contact $C_{N-1,1}$ with $N>$ 2 is $N-1$ times larger than $C_{1,1}$ in the high temper- 


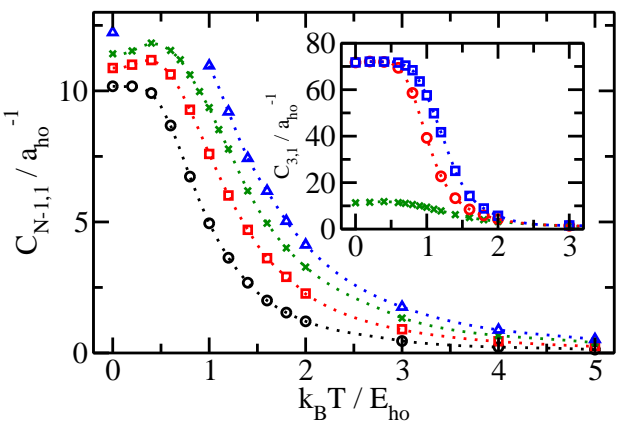

FIG. 3: (Color online) Circles, squares, crosses and triangles show $C_{N_{1}, N_{2}}$ for the $(1,1),(2,1),(3,1)$ and $(4,1)$ systems, respectively, interacting through $V_{\mathrm{G}}$ with $r_{0}=0.06 a_{\mathrm{ho}}$ as a function of $T$ [39]. Dotted lines serve as a guide to the eye. Inset: Crosses, squares and circles show the contact of the $(3,1)$ system for Fermi, Boltzmann and Bose statistics, respectively.

ature limit. In the low temperature limit (see Fig. 3), in contrast, $C_{N-1,1}$ with $N>2$ is only slightly larger than $C_{1,1}$, reflecting the fact that, to leading order, the system can form one and not $N-1$ bound pairs. It is also interesting to compare the limiting behaviors of $C_{3,1}$ and $C_{2,2} . C_{2,2}$ is $4 / 3$ times larger than $C_{3,1}$ at large $T$ [see Eq. (4)] but roughly two times larger at low $T$. The latter reflects the fact that the $(2,2)$ and $(3,1)$ systems can form two dimers and one dimer, respectively.

To elucidate the role of the Fermi statistics, we focus on the $(3,1)$ system at unitarity with $r_{0}=0.06 a_{\mathrm{ho}}$. The inset of Fig. 3 shows the contact obtained by treating the majority particles as identical fermions (crosses; these are the same data as discussed above), as identical bosons (circles) and as distinguishable "Boltzmann particles" (squares). In the high temperature regime, the Fermi and Bose statistics can be treated as a correction to the Boltzmann statics. In the low temperature regime, in contrast, appreciable differences are revealed. The $(3,1)$ systems with Bose and Boltzmann statistics share the same ground state and thus the same contact in the zero temperature limit. Compared to the contact of the Bose and Boltzmann systems, that of the Fermi system is strongly suppressed as a consequence of the Pauli exclusion principle. Specifically, the $(3,1)$ Fermi system at unitarity does not support a bound state in free space while the $(3,1)$ Bose and Boltzmann systems do. The existence of self-bound states leads to an increased amplitude of the pair distribution function at small interspecies distances. Moreover, the Bose and Boltzmann systems are - unlike the Fermi system - not fully universal, i.e., their properties are, in addition to the $s$-wave scattering length, governed by a three-body parameter. This implies that the Bose and Boltzmann systems are characterized by a non-zero three-body contact in addition to the two-body contact considered throughout this paper [40, 41].

Finite-temperature effects play an important role in many finite-sized systems, including atomic clusters, nuclei and quantum dots. Our work demonstrates that small harmonically trapped two-component Fermi gases with infinitely strong interspecies $s$-wave interactions, which can be realized and probed experimentally, also exhibit intriguing dependencies on the temperature. In particular, we proposed a high-temperature cluster expansion in the canonical ensemble, quantified the range dependence of the contact, observed and interpreted the distinctly different behavior of the contact of spinbalanced and spin-imbalanced Fermi gases in the low temperature regime, and elucidated the role of the Fermi statistics. Throughout, we reported the temperature in terms of the natural energy scale of the harmonic oscillator. Other relevant temperature scales are the Fermi temperature $T_{F}$ and the critical temperature $T_{c}$, $T_{F}=2.5 E_{\mathrm{ho}} / k_{B}$ for $N=3-5\left[42\right.$ and $T_{c} \approx 0.2 T_{F}$ for the trapped spin-balanced system [43]. Our calculations cover temperatures much smaller and much larger than these characteristic temperature scales. Future studies will be aimed at determining the critical temperature, and the superfluid fraction and superfluid density of small trapped Fermi gases.

Support by the National Science Foundation (NSF) through Grant No. PHY-1205443 is gratefully acknowledged. This work used the Extreme Science and Engineering Discovery Environment (XSEDE), which is supported by NSF grant number OCI-1053575, and the WSU HPC. This work was additionally supported by the NSF through a grant for the Institute for Theoretical Atomic, Molecular and Optical Physics at Harvard University and Smithsonian Astrophysical Observatory.
[1] S. Tan, Ann. Phys. 323, 2952 (2008).

[2] S. Tan, Ann. Phys. 323, 2971 (2008).

[3] S. Tan, Ann. Phys. 323, 2987 (2008).

[4] F. Werner and Y. Castin, Phys. Rev. A 86, 013626 (2012).

[5] E. Braaten and L. Platter, Phys. Rev. Lett. 100, 205301 (2008).

[6] J. T. Stewart, J. P. Gaebler, T. E. Drake, and D. S. Jin,
Phys. Rev. Lett. 104, 235301 (2010).

[7] E. D. Kuhnle, H. Hu, X.-J. Liu, P. Dyke, M. Mark, P. D. Drummond, P. Hannaford, and C. J. Vale, Phys. Rev. Lett. 105070402 (2010).

[8] E. D. Kuhnle, S. Hoinka, P. Dyke, H. Hu, P. Hannaford, and C. J. Vale, Phys. Rev. Lett. 106170402 (2011).

[9] Y. Sagi, T. E. Drake, R. Paudel, and D. S. Jin, Phys. Rev. Lett. 109, 220402 (2012). 
[10] S. Hoinka, M. Lingham, K. Fenech, H. Hu, C. J. Vale, J. E. Drut, and S. Gandolfi, Phys. Rev. Lett. 110, 055305 (2013).

[11] D. Blume and K. M. Daily, Phys. Rev. A 80, 053626 (2009)

[12] F. Werner, L. Tarruell, and Y. Castin, Eur. Phys. J. B 68, 401 (2009).

[13] F. Palestini, A. Perali, P. Pieri, and G. C. Strinati, Phys. Rev. A 82, 021605(R) (2010).

[14] J. E. Drut, T. A. Lähde, and T. Ten, Phys. Rev. Lett. 106, 205302 (2011).

[15] K. Van Houcke, F. Werner, E. V. Kozik, N. V. Prokof'ev, and B. V. Svistunov, arXiv:1303.6245.

[16] I. Boettcher, S. Diehl, J. M. Pawlowski, and C. Wetterich, Phys. Rev. A 87, 023606 (2013).

[17] M. Barth and W. Zwerger, Ann. Phys. 326, 2544 (2011).

[18] E. V. H. Doggen and J. J. Kinnunen, arXiv:1304.0918

[19] T.-L. Ho and E. J. Mueller, Phys. Rev. Lett. 92, 160404 (2004).

[20] X.-J. Liu, H. Hu, and P. D. Drummond, Phys. Rev. Lett. 102, 160401 (2009).

[21] X.-J. Liu, Phys. Rep. 524, 37 (2013).

[22] K. Huang and C. N. Yang, Phys. Rev. 105, 767 (1957).

[23] T. Busch, B.-G. Englert, K. Rzążzewski, and M. Wilkens, Found. Phys. 28, 549 (1998).

[24] F. Werner and Y. Castin, Phys. Rev. Lett. 97, 150401 (2006).

[25] Y. Suzuki and K. Varga. Stochastic Variational Approach to Quantum Mechanical Few-Body Problems. Springer Verlag, Berlin (1998).

[26] K. M. Daily and D. Blume, Phys. Rev. A 81, 053615 (2010).

[27] D. Rakshit, K. M. Daily, and D. Blume, Phys. Rev. A 85, 033634 (2012).

[28] D. M. Ceperley, Rev. Mod. Phys. 67, 279 (1995).

[29] see, e.g., D. M. Ceperley, J. Stat. Phys. 63, 1237 (1991).

[30] K. M. Daily, X. Y. Yin, and D. Blume, Phys. Rev. A 85,
053614 (2012).

[31] See the supplemental material at www.to.be.inserted.by.the.editor for details.

[32] J. P. Kestner and L.-M. Duan, Phys. Rev. A 76, 033611 (2007).

[33] I. Stetcu, B. R. Barrett, U. van Kolck, and J. P. Vary, Phys. Rev. A 76, 063613 (2007).

[34] J. von Stecher, D. Blume, and C. H. Greene, Phys. Rev. A 77, 043619 (2008).

[35] E. Pulvirenti and D. Tsagkarogiannis, Commun. Math. Phys. 316, 289 (2012).

[36] S. Giorgini, L. P. Pitaevskii, and S. Stringari, Rev. Mod. Phys. 80, 1215 (2008).

[37] D. Blume, Rep. Prog. Phys. 75, 046401 (2012).

[38] H. Hu, X.-J. Liu, and P. D. Drummond, New J. Phys. 13, 035007 (2011).

[39] For the $(4,1)$ system, the results derived from the basis set expansion approach cover the temperature range $k_{B} T \lesssim 0.1 E_{\mathrm{ho}}$. The results derived from the PIMC approach cover the temperature range $k_{B} T \gtrsim E_{\mathrm{ho}}$. For this system, we did not treat the temperature regime between $0.1 E_{\mathrm{ho}} \lesssim k_{B} T \lesssim E_{\mathrm{ho}}$. Nevertheless, our results suggest that $C_{4,1}$ exhibits a maximum at finite $T$, like the other maximally polarized systems considered in this paper.

[40] The three-body contact [41] should not be confused with the two-body contact $C_{N_{1}, N_{2}}$ of the two-, three- and higher-body systems. For the Fermi systems considered in this work, the three-body contact vanishes identically.

[41] E. Braaten, D. Kang and L. Platter, Phys. Rev. Lett. 106, 153005 (2011).

[42] The Fermi temperature of small harmonically trapped two-component Fermi gases is defined through the energy of the highest single-particle state of the non-interacting system.

[43] L. A. Sidorenkov, M. K. Tey, R. Grimm, Y.-H. Hou, L. Pitaevskii, and S. Stringari, arXiv:1302.2871. 


\title{
Supplemental material for "Harmonically trapped Fermi gas: Temperature dependence of the Tan contact"
}

\author{
Yangqian Yan $^{1}$ and D. Blume ${ }^{1,2}$ \\ ${ }^{1}$ Department of Physics and Astronomy, Washington State University, Pullman, Washington 99164-2814, USA \\ ${ }^{2}$ ITAMP, Harvard-Smithsonian Center for Astrophysics, Cambridge, Massachusetts 02138, USA
}

(Dated: July 19, 2018)

PACS numbers:

The notation used in this supplemental material follows that introduced in the main text.

\section{$(1,1)$ system with zero-range interactions}

The relative energies of the $(1,1)$ system with $s$-wave zero-range interactions can be determined by solving the transcendental equation [1]

$$
\frac{\sqrt{2} \Gamma\left(3 / 4-E^{\mathrm{rel}} /\left(2 E_{\mathrm{ho}}\right)\right)}{\Gamma\left(1 / 4-E^{\mathrm{rel}} /\left(2 E_{\mathrm{ho}}\right)\right)}=\frac{a_{\mathrm{ho}}}{a_{s}} .
$$

At unitarity, the relative $s$-wave energies read $E_{j}^{\text {rel }}=$ $(2 j+1 / 2) E_{\mathrm{ho}}$, where $j=0,1, \cdots$. States with relative orbital angular momentum $L$ greater than zero are not affected by the interactions. Using these energies and the expressions for the change of the relative energies with $-1 / a_{s}$ from the main text, one finds

$$
C_{1,1}(\tilde{\omega})=8 \sqrt{\pi} \frac{e^{\tilde{\omega}}\left(e^{\tilde{\omega}}-1\right)^{2} \sqrt{e^{-\tilde{\omega}} \sinh (\tilde{\omega})}}{e^{\tilde{\omega}}\left[e^{\tilde{\omega}}\left(e^{\tilde{\omega}}-2\right)+4\right]-1} a_{\mathrm{ho}}^{-1}
$$

[see dotted line in Fig. 2(a) of the main text].

To quantify the corrections that arise from the finite range of the interspecies interaction potential, we consider three approaches:

(i) Using a B-spline approach, we calculate the relative energies of the $(1,1)$ system as a function of $-1 / a_{s}$ up to $500 E_{\mathrm{ho}}$ for the first 50 angular momentum channels. Using these energies and the corresponding slopes, we calculate the contact numerically. The solid line in Fig. 2(a) of the main text shows the result for the Gaussian potential with $r_{0}=0.06 a_{\text {ho }}$.

(ii) We replace $-1 / a_{s}$ in Eq. (11) by $-1 / a_{s}+r_{\text {eff }} k^{2} / 2$, where $r_{\text {eff }}$ denotes the effective range and $k$ is related to the relative energy via $\hbar^{2} k^{2} / m=E^{\text {rel }}$. The leading order effective range corrections to the energy and to the change of the energy at unitarity can then be derived analytically [2]. Using these expressions, the contact can be readily determined numerically within the microscopic approach. On the scale of Fig. 2(a) of the main text, the resulting contact would be indistinguishable from the solid line.

(iii) To account for the fact that higher partial wave channels are affected by the interspecies interactions if

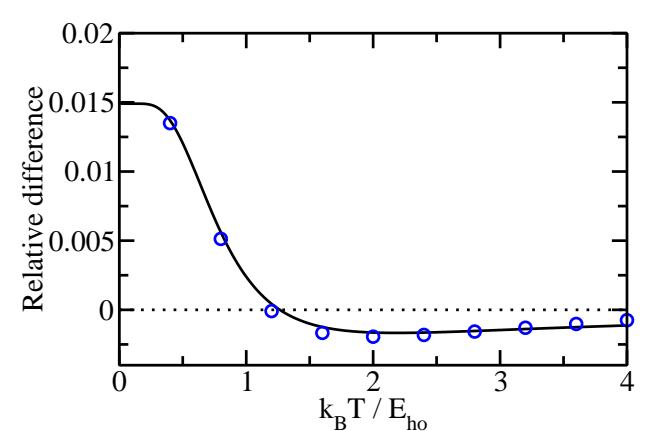

FIG. 1: (Color online) Range dependence of the contact $C_{1,1}$ for $r_{0}=0.06 a_{\mathrm{ho}}$ as a function of $k_{B} T / E_{\mathrm{ho}}$. The circles show the difference between the finite-range contact calculated using approach (i) and the zero-range contact, normalized by the zero-range contact. The solid line shows the difference between the finite-range contact calculated using approach (ii) and the zero-range contact, normalized by the zero-range contact. In the large $T$ limit, the difference approaches zero from below. In approach (ii), $r_{\text {eff }}=0.12178 a_{\mathrm{ho}}$-corresponding to $V_{\mathrm{G}}$ with $r_{0}=0.06 a_{\mathrm{ho}}$ - has been used.

the range of the underlying two-body potential is finite, we generalize the above effective range treatment to finite angular momenta. Specifically, we replace the generalized scattering lengths in the transcendental equations for the higher partial waves [3] by the corresponding effective range expansions and determine the corrections to the energy and to the slope of the energy for vanishing generalized scattering lengths due to the effective range. Calculating the effective ranges for the Gaussian potential with $r_{0}=0.06 a_{\text {ho }}$ for the lowest few partial wave channels, we find that the effective range correction of the contact due to the $L=1$ channel is about $0.0003 \%$ and $0.015 \%$ for $k_{B} T / E_{\mathrm{ho}}=1 / 2$ and 2 , respectively. For fixed $T$, the corrections decrease with increasing $L$. Although the $L=1$ channel leads to a larger percentage correction at large $T$ than at small $T$, its overall role is negligible since the contact itself is small in the large $T$ regime.

Overall, the agreement between the contacts calculated using approaches (i)-(iii) is excellent. To bring out the size of the finite-range corrections, Fig. 1 shows the difference between the finite-range and zero-range con- 
tacts, normalized by the zero-range contact. The circles show the relative difference for the case where the finiterange contact is calculated using approach (i) while the solid line shows the relative difference for the case where the finite-range contact is calculated using approach (ii). The difference is largest at low $T$ and decreases rapidly. The difference changes sign at $k_{B} T \approx 1.25 E_{\mathrm{ho}}$ and then approaches zero from the negative side. For $k_{B} T \gtrsim E_{\mathrm{ho}}$, the percentage deviation is smaller than $0.5 \%$ and thus, for all practical purposes, negligible.

A key result of the above analysis is that the two-body contact is nearly fully determined by the $s$-wave channel and that higher partial wave contributions play a negligible role if the interspecies interactions are modeled by the Gaussian potential $V_{\mathrm{G}}$ with $r_{0}=0.06 a_{\mathrm{ho}}$. This is crucial for our analysis of the $(2,1)$ system discussed in the next section, which assumes interspecies $s$-wave zero-range interactions. It also suggests that our high temperature results for larger systems, obtained by using $V_{\mathrm{G}}$ with $r_{0}=0.06 a_{\mathrm{ho}}$, are very close to the universal zero-range results.

\section{$(2,1)$ system with zero-range interactions}

To determine the contact of the $(2,1)$ system with zerorange interactions at unitarity, we resort to the hyperspherical coordinate approach [4, 5]. In this approach, the solutions of the relative Schrödinger equation are obtained in a two step process. First, the hyperangular Schrödinger equation is solved for fixed hyperradius $R$. Second, a set of coupled hyperradial Schrödinger equations is solved. For zero-range interactions with infinitely large $s$-wave scattering length, the coupling between the hyperangular and hyperradial degrees of freedom vanishes and the relative eigen energies at unitarity can be written as $E^{\mathrm{rel}}=\left(2 q+s_{L, \nu}+1\right) E_{\mathrm{ho}}$, where the hyperradial quantum number $q$ takes the values $0,1, \cdots$. The $s_{L, \nu}$ are solutions of the transcendental equation [4, 5]

$$
\begin{array}{r}
\frac{(-1)^{L}{ }_{2} F_{1}\left(\frac{1}{2}\left(L-s_{L, \nu}+2\right), \frac{1}{2}\left(L+s_{L, \nu}+2\right) ; L+\frac{3}{2} ; \frac{1}{4}\right)}{\pi(2 L+1) ! !}+ \\
\frac{1}{\Gamma\left(\frac{1}{2}\left(L-s_{L, \nu}+1\right)\right) \Gamma\left(\frac{1}{2}\left(L+s_{L, \nu}+1\right)\right)} \\
=\frac{1}{\sqrt{2} \Gamma\left(\frac{1}{2}\left(L-s_{L, \nu}+2\right)\right) \Gamma\left(\frac{1}{2}\left(L+s_{L, \nu}+2\right)\right)} \frac{R}{a_{s}}
\end{array}
$$

for $1 / a_{s}=0$. In Eq. (3), the hyperradius $R$ is defined through $R^{2}=2\left(r_{12}^{2}+r_{23}^{2}+r_{13}^{2}\right) / 3$ and ${ }_{2} F_{1}$ denotes the hypergeometric function.

To determine the change of the relative energies at unitarity with $-1 / a_{s}$, we replace $s_{L, \nu}$ in Eq. (3) by $s_{L, \nu}+\Delta s_{L, \nu}$, where $\left|\Delta s_{L, \nu}\right|$ is assumed to be small. Taylor expanding Eq. (3) around the known $s_{L, \nu}$ value, we find $\Delta s_{L, \nu}=c_{L, \nu} R / a_{s}$. We insert this into the effective hyperradial potential $\hbar^{2}\left[\left(s_{L, \nu}+\Delta s_{L, \nu}\right)^{2}-1 / 4\right] /\left(2 m R^{2}\right)$ and treat the leading order correction as a perturbation, i.e., we define $V_{\text {pert }}(R)=\hbar^{2} c_{L, \nu} s_{L, \nu} /\left(m R a_{s}\right)$. For each $s_{L, \nu}$, we calculate the exact proportionality constant $c_{L, \nu}$ and determine the change of the relative energy by treating $V_{\text {pert }}(R)$ in first-order perturbation theory in the hyperradial Schrödinger equation, i.e., by evaluating the integral $\int_{0}^{\infty}\left|F_{q, s_{L, \nu}}(R)\right|^{2} V_{\text {pert }}(R) d R$. The unperturbed radial wave functions $F_{q, s_{L, \nu}}(R)$ are obtained by solving the hyperradial Schrödinger equation for the unitary problem. Using the known expressions for $F_{q, s_{L}, \nu}(R)$ [4], the integral can be evaluated analytically, yielding the leading-order variation of $E^{\text {rel }}$ with $-1 / a_{s}$ for each $s_{L, \nu}$ and $q=0,1, \cdots$. The approach outlined here reproduces the recurrence relations of Refs. [6, 7]. Using Mathematica, the energies and slopes of the energies of the $(2,1)$ system at unitarity can be calculated with essentially arbitrary accuracy, thereby allowing us to calculate the temperature-dependent contact with high accuracy. In calculating the partition function, care has to be exercised as the above formalism excludes a large number of "trivial energy levels" that are not affected by the $s$-wave interactions [4]. We account for these "trivial states" using the ideas discussed in Ref. [8].

Table $\llbracket$ tabulates the contact $C_{2,1}$ [see also dotted line in Fig. 2(b) of the main text]. These zero-range results serve as a benchmark for our PIMC simulations. Moreover, the semi-analytic expressions for the energy and its variation with $-1 / a_{s}$ can also be used to calculate the third-order contact coefficient [9]. We find 0.0269223(3), which notably improves upon the accuracy of the value of 0.02692(2) of Ref. [9]; a more detailed discussion of the connection between the second- and third-order contact coefficients [9] and our cluster expansion is given below.

We now refine the two-state model, which was used in the main text to explain why the maximum of $C_{2,1}$ occurs at finite $T$. Circles in Fig. 2 show the zero-temperature contacts $\bar{C}_{2,1}\left(E_{j}^{\mathrm{rel}}\right), \bar{C}_{2,1}\left(E_{j}^{\mathrm{rel}}\right)=$ $\left(4 \pi m / \hbar^{2}\right) \partial E_{j}^{\text {rel }} /\left(\partial\left(-a_{s}^{-1}\right)\right)$, associated with the $j$ th eigenstate of the $(2,1)$ system at unitarity as a function of $E^{\text {rel }}$. Panel (a) focuses on the small $E^{\text {rel }}$ region while panel (b) extends up to $E^{\text {rel }}=30 E_{\mathrm{ho}}$. Figure 2 shows that the zero-temperature contacts are non-negative. Moreover, all $\bar{C}_{2,1}\left(E_{j}^{\text {rel }}\right)$ but one are smaller than $11 a_{\mathrm{ho}}^{-1}$. The "outlier" corresponds to the lowest $L=0$ state, (3) i.e., the state with the second lowest eigen energy. To calculate the temperature-dependent contact $C_{2,1}$ within the microscopic approach, the zero-temperature contacts $\bar{C}_{2,1}\left(E_{j}^{\text {rel }}\right)$ need to be weighted by the temperaturedependent Boltzmann factors $\exp \left[-E_{j}^{\mathrm{rel}} /\left(k_{B} T\right)\right] / Z^{\mathrm{rel}}(T)$, where $Z^{\text {rel }}(T)$ denotes the partition function that accounts for the relative degrees of freedom. Lines in Fig. 22(a) show these "weight factors" for four different $T$, $k_{B} T / E_{\mathrm{ho}}=0.2,0.4,0.6$ and 0.8 . It can be seen that the weight factor drops off quickly for $k_{B} T / E_{\mathrm{ho}}=0.2$, indicating that the "outlier" as well as the zero-temperature 
TABLE I: Contact $C_{2,1}$ as a function of temperature for $s$ wave zero-range interactions at unitarity.

\begin{tabular}{c|c}
\hline \hline$k_{B} T / E_{\mathrm{ho}}$ & $C_{2,1} / a_{\mathrm{ho}}^{-1}$ \\
\hline 0.1 & 10.4986 \\
0.2 & 10.6717 \\
0.3 & 10.8225 \\
0.4 & 10.8529 \\
0.5 & 10.7047 \\
0.6 & 10.3479 \\
0.7 & 9.79272 \\
0.8 & 9.08421 \\
0.9 & 8.28504 \\
1.0 & 7.45680 \\
1.1 & 6.64831 \\
1.2 & 5.89176 \\
1.3 & 5.20435 \\
1.4 & 4.59212 \\
1.5 & 4.05395 \\
1.6 & 3.58469 \\
1.7 & 3.17735 \\
1.8 & 2.82444 \\
1.9 & 2.51872 \\
2.0 & 2.25359 \\
2.1 & 2.02320 \\
2.2 & 1.82248 \\
2.3 & 1.64710 \\
2.4 & 1.49338 \\
2.5 & 1.35820 \\
2.6 & 1.23893 \\
2.7 & 1.13335 \\
2.8 & 1.03959 \\
2.9 & 0.956067 \\
3.0 & 0.881422 \\
3.1 & 0.814516 \\
3.2 & 0.754375 \\
3.3 & 0.700163 \\
3.4 & 0.651166 \\
3.5 & 0.606768 \\
3.6 & 0.566439 \\
3.7 & 0.529719 \\
3.8 & 0.496209 \\
3.9 & 0.465562 \\
4.0 & 0.437476 \\
4.1 & 0.411684 \\
4.2 & 0.387954 \\
4.3 & 0.366081 \\
4.4 & 0.345884 \\
4.5 & 0.327202 \\
4.6 & 0.309894 \\
4.7 & 0.293834 \\
4.8 & 0.278908 \\
\hline \hline & 0.265017 \\
\hline
\end{tabular}

contacts of the other excited states contribute negligibly to $C_{2,1}$. At $k_{B} T / E_{\mathrm{ho}}=0.4$, in contrast, the "outlier" carries appreciable weight compared to the zerotemperature contact of the lowest eigen state. At yet higher $T$, the weight factor falls off slower, thereby reducing the relative importance of the "outlier".

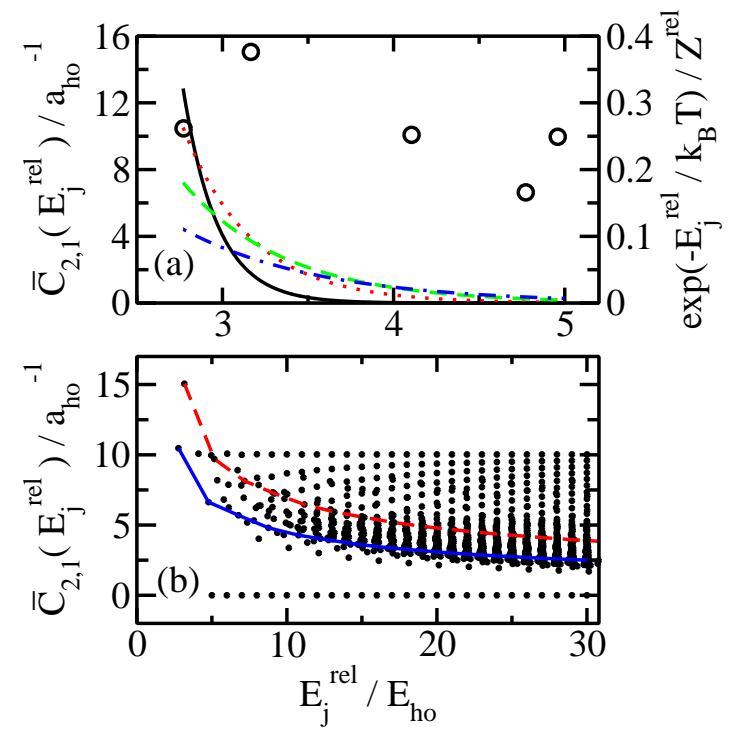

FIG. 2: (Color online) (a) The solid, dotted, dashed and dashdotted lines show the weight factor $\exp \left[-E_{j}^{\text {rel }} /\left(k_{B} T\right)\right] / Z^{\text {rel }}$ (see right axis) as a function of the relative energy for $k_{B} T / E_{\mathrm{ho}}=0.2,0.4,0.6$ and 0.8 , respectively. The circles show the zero-temperature contact $\bar{C}_{2,1}$ (see left axis) for states with $E_{j}^{\text {rel }}<5 E_{\text {ho }}$. (b) The dots show the zerotemperature contact $\bar{C}_{2,1}$ as a function of the relative energy for $E_{j}^{\text {rel }} \lesssim 30 E_{\text {ho. }}$ The solid (dashed) line connects the contacts of $\tilde{L}=1(L=0)$ states, which are characterized by the same $s_{L, \nu}$ value but different $q$ values; the $s_{L, \nu}$ values chosen are the smallest ones for both $L=1$ and 0 .

\section{Basis set expansion approach}

The explicitly correlated Gaussian basis set expansion approach with semi-stochastic parameter optimization has been used extensively in the literature to describe the behavior of small harmonically trapped twocomponent Fermi gases with short-range interactions (see, e.g., Ref. [10] for details). The energies of the threeand four-body systems can be calculated with an accuracy of better than $1 \%$ (and often better than $0.1 \%$ ). To determine the solid lines in Figs. 2(b)-2(d) of the main text, we used energy cutoffs $E^{\text {rel }}$ around $11 E_{\mathrm{ho}}, 9 E_{\mathrm{ho}}$, and $9.5 E_{\mathrm{ho}}$, respectively. Including the degeneracies arising from the projection quantum number as well as the relative energies of states that are only very weakly affected by the short-range interactions, this amounts to around 1250, 230 and 700 energy levels for the $(2,1)$, $(3,1)$ and $(2,2)$ systems, respectively. The use of a finite energy cutoff implies that the determination of the finite-range contact within the microscopic approach is limited to the low temperature regime. Convergence of the contact with respect to the energy cutoff was tested by including successively fewer energy levels. 
TABLE II: High-temperature expansion coefficients $c_{1,1}^{(i)}$ and $c_{2,1}^{(i)}$ for the cluster expansion in the canonical ensemble.

\begin{tabular}{c|ccccc}
\hline \hline$i$ & 0 & 1 & 2 & 3 & 4 \\
\hline$c_{1,1}^{(i)}$ & $4 \sqrt{\pi}$ & 0 & $\sqrt{\pi} / 6$ & $-2 \sqrt{\pi}$ & $\sqrt{\pi} / 96$ \\
$c_{2,1}^{(i)}$ & & & & $-7.012(12)$ & $0.0(1)$ \\
\hline \hline
\end{tabular}

\section{Path integral Monte Carlo approach}

Our PIMC implementation largely follows that described in Refs. [11, 12]. In the low temperature regime, we find that we have to adjust the simulation parameters that control the sampling of the unpermuted configurations as well as those that control the sampling of the permutations carefully. We employ the second- and fourthorder Trotter formula [13, 14]; higher-order propagation schemes did not seem to lead to further improvements.

It is well known that the standard PIMC algorithm, as employed here, suffers from the Fermi sign problem [15]. Simply put, the signal to noise ratio decreases exponentially with decreasing temperature and increasing number of particles. As demonstrated by our simulation results, the Fermi sign problem is sufficiently small for the parameter region considered in this Letter. In particular, the PIMC algorithm allows us to investigate a region of the physical parameter space (strong short-range interactions and relatively low temperature) that is inaccessible by other numerical approaches.

\section{Cluster expansion in canonical ensemble and connection to virial equation of state in grandcanonical ensemble}

This section elaborates on the cluster expansion introduced in the main part of the text. To further explore the high temperature behavior, we Taylor expand $C_{1,1}$ and $C_{2,1}-2 C_{1,1}$ around $\tilde{\omega} \ll 1$. We find

$$
C_{1,1}=\tilde{\omega}^{5 / 2} \sum_{i=0}^{\infty} c_{1,1}^{(i)} \tilde{\omega}^{i} a_{\mathrm{ho}}^{-1}
$$

and

$$
C_{2,1}-2 C_{1,1}=\tilde{\omega}^{5 / 2} \sum_{i=3}^{\infty} c_{2,1}^{(i)} \tilde{\omega}^{i} a_{\mathrm{ho}}^{-1}
$$

Table II lists the dimensionless coefficients $c_{1,1}^{(i)}$ and $c_{2,1}^{(i)}$ for $i \leq 4$.

We now connect the high temperature cluster expansion in the canonical ensemble to the more frequently used high-temperature virial expansion in the grand canonical ensemble [16], which assumes large number of particles. For simplicity, we consider spin-balanced harmonically trapped systems, i.e., systems with $N_{1}=N_{2}=$
$N / 2$. The contact $C_{\mathrm{gc}}$ in the grand canonical virial expansion reads [9]

$C_{\mathrm{gc}}=2^{7 / 2} \pi^{3 / 2} \tilde{\omega}^{-1 / 2} Z_{1}(\tilde{\omega})\left[c_{2}(\tilde{\omega}) z^{2}+c_{3}(\tilde{\omega}) z^{3}+\cdots\right] a_{\mathrm{ho}}^{-1}$,

where $Z_{1}(\tilde{\omega})$ denotes the partition function of a single particle in a spherically symmetric harmonic trap and $z$ the fugacity, $z=\exp \left(\mu / k_{B} T\right)$; here, $\mu$ is the chemical potential. The contact coefficients $c_{2}(\tilde{\omega})$ and $c_{3}(\tilde{\omega})$ for the trapped system depend on the temperature and can be derived from the second- and third-order virial coefficients of the trapped system [9]. In the following, we use the high temperature expansions

$$
c_{2}(\tilde{\omega})=\frac{1}{2 \sqrt{2} \pi}\left(1-\frac{\tilde{\omega}^{2}}{12}+\cdots\right)
$$

and

$$
c_{3}(\tilde{\omega})=0.0269223(3)+\cdots
$$

The high-temperature expansion of the partition function reads

$$
Z_{1}(\tilde{\omega})=\tilde{\omega}^{-3}\left(1-\frac{\tilde{\omega}^{2}}{8}+\cdots\right)
$$

The fugacity $z$ can be determined from the number equation [9]. Expanding the number equation for small $z$, we find

$$
z=\frac{N}{2}\left(\tilde{\omega}^{3}+\frac{\tilde{\omega}^{5}}{8}-\frac{3}{8} \frac{N}{2} \tilde{\omega}^{6}+\cdots\right) .
$$

Inserting the expansions given in Eqs. (77)-(10) into Eq. (6), we find

$$
\begin{array}{r}
C_{\mathrm{gc}}=\left(4 \sqrt{\pi} \frac{N^{2}}{4} \tilde{\omega}^{5 / 2}+\frac{\sqrt{\pi}}{6} \frac{N^{2}}{4} \tilde{\omega}^{9 / 2}-3 \sqrt{\pi} \frac{N^{3}}{8} \tilde{\omega}^{11 / 2}\right. \\
\left.-1.69606(2) \frac{N^{3}}{8} \tilde{\omega}^{11 / 2}+\cdots\right) a_{\mathrm{ho}}^{-1} .(11)
\end{array}
$$

To relate Eq. (11) to Eqs. (4) and (5), we write, in analogy with Eq. (4) of the main text,

$$
\frac{C_{\mathrm{gc}}}{N^{2} / 4}=C_{\mathrm{gc} ; 1,1}+\frac{N-2}{2}\left(C_{\mathrm{gc} ; 2,1}-2 C_{\mathrm{gc} ; 1,1}\right)+\cdots,
$$

where

$$
C_{\mathrm{gc} ; 1,1}=\tilde{\omega}^{5 / 2} \sum_{i=0}^{\infty} c_{\mathrm{gc} ; 1,1}^{(i)} \tilde{\omega}^{i} a_{\mathrm{ho}}^{-1}
$$

and

$$
C_{\mathrm{gc} ; 2,1}-2 C_{\mathrm{gc} ; 1,1}=\tilde{\omega}^{5 / 2} \sum_{i=3}^{\infty} c_{\mathrm{gc} ; 2,1}^{(i)} \tilde{\omega}^{i} a_{\mathrm{ho}}^{-1}
$$

The coefficients $c_{\mathrm{gc} ; 1,1}^{(i)}$ and $c_{\mathrm{gc} ; 2,1}^{(i)}$ are listed in Table III. Comparison of Tables II] and III shows that the coeffi- 
TABLE III: High-temperature expansion coefficients $c_{\mathrm{gc} ; 1,1}^{(i)}$ and $c_{\mathrm{gc} ; 2,1}^{(i)}$ extracted from the contact determined in the grand canonical ensemble.

\begin{tabular}{c|cccc}
\hline \hline$i$ & 0 & 1 & 2 & 3 \\
\hline$c_{\mathrm{gc} ; 1,1}^{(i)}$ & $4 \sqrt{\pi}$ & 0 & $\sqrt{\pi} / 6$ & $-7.01342(2)$ \\
$c_{\mathrm{gc} ; 2,1}^{(i)}$ & & & & $-7.01342(2)$ \\
\hline \hline
\end{tabular}

cients with $i=0-2$ agree but that discrepancies exist for $i=3$. Specifically, our analysis shows that the leading order three-body coefficients agree, i.e., $c_{\mathrm{gc} ; 2,1}^{(3)}=c_{2,1}^{(3)}$, but that the two-body term at the same order in $\tilde{\omega}$ does not agree, i.e., $c_{\mathrm{gc} ; 1,1}^{(3)} \neq c_{1,1}^{(3)}$. Disagreement is expected since the canonical and grand canonical ensembles are known to yield different results. The fact that the disagreement appears in the term proportional to $N^{2}$ and not the term proportional to $N^{3}$ makes sense, since our analysis in the grand canonical ensemble assumes large $N$, rendering the term proportional to $N^{2}$ less important than the term proportional to $N^{3}$.

[1] T. Busch, B.-G. Englert, K. Rzążewski, and M. Wilkens, Found. Phys. 28, 549 (1998).
[2] K. M. Daily, X. Y. Yin, and D. Blume, Phys. Rev. A 85, 053614 (2012).

[3] R. Stock, A. Silberfarb, E. L. Bolda, and I. H. Deutsch, Phys. Rev. Lett. 94, 023202 (2005).

[4] F. Werner and Y. Castin, Phys. Rev. Lett. 97, 150401 (2006).

[5] S. T. Rittenhouse, N. P. Mehta, and C. H. Greene, Phys. Rev. A 82, 022706 (2010).

[6] F. Werner and Y. Castin, Phys. Rev. A 86, 013626 (2012).

[7] S. Moroz, Phys. Rev. A 86, 011601 (2012).

[8] K. M. Daily and D. Blume, Phys. Rev. A 81, 053615 (2010).

[9] H. Hu, X.-J. Liu, and P. D. Drummond, New J. Phys. 13, 035007 (2011).

[10] D. Rakshit, K. M. Daily, and D. Blume, Phys. Rev. A 85, 033634 (2012).

[11] D. M. Ceperley, Rev. Mod. Phys. 67, 279 (1995).

[12] M. Boninsegni, J. Low Temp. Phys. 141, 27 (2005).

[13] S. A. Chin, Phys. Lett. A 226, 344 (1997).

[14] S. Jang, S. Jang and G. A. Voth, J. Chem. Phys. 115, 7832 (2001).

[15] see, e.g., D. M. Ceperley, J. Stat. Phys. 63, 1237 (1991).

[16] K. Huang, Statistical Mechanics, 2nd Ed. (John Wiley and Sons, Inc., New York, 1963). 